\title{
Common fixed point theorems for several multivalued mappings on proximinal sets in regular modular space
}

\author{
N. Faried ${ }^{1}$, H. Abd El-Ghaffar ${ }^{1}$ and S. Hamdy ${ }^{1 *}$
}

"Correspondence:

solihamdy123@gmail.com

${ }^{1}$ Faculty of Science, Ain Shams

University, 11566 Abbassia, Cairo,

Egypt

\begin{abstract}
In this paper, we found a common fixed point for several multivalued mappings on proximinal sets in regular modular metric space. Also, we introduced the notions of conjoint F-proximinal contraction as well as conjoint F-proximinal contraction of Hardy-Rogers-type for several multivalued mappings. Furthermore, we enhanced our results by giving an application in integral equations.
\end{abstract}

Keywords: Multivalued mappings; Conjoint F-contraction; $\Delta_{2}$-condition and $\Delta_{M}$-Condition

\section{Introduction}

In 2010, the notion of modular metric space was introduced by Chistyakov [3]. In 2012, Wardowski characterized the idea of F-contraction which generalized the Banach contraction principle in various manners and he utilized the new concept of contraction to find the fixed point theorem [15]. Also, Mongkolkeha et al. proved the existence of common fixed points for a generalized weak contractive mapping in modular spaces. Moreover, they proved the existence of some fixed point theorems without the $\Delta_{2}$-condition [11].

Furthermore, in 2013, Sgroi et al. achieved a multivalued version of Wardowski's result [14].

In 2014, Abdou et al. studied the existence of fixed points for contractive-type multivalued maps in the setting of modular metric spaces [1].

In 2015, Rahimpoor et al. generalized and extended results of Mongkolkeha et al. [11] by proving some coincidence and common fixed point theorems for a contractive mapping in modular metric spaces [13].

Also, in 2016, Dilip Jain et al. presented multivalued F-contraction in the case of modular metric space with specific assumptions [7]. These results were an extension of Nadler, Wardowski, and Sgroi to the case of modular metric spaces [12, 14, 15].

In 2018, Khan et al. presented a common fixed point theorem for a pair of multivalued F- $\Psi$-proximinal mappings satisfying Ciric-Wardowski-type contraction in partial metric spaces. Also, they introduced an example and application to system of integral equations [10].

(c) The Author(s) 2021. This article is licensed under a Creative Commons Attribution 4.0 International License, which permits use sharing, adaptation, distribution and reproduction in any medium or format, as long as you give appropriate credit to the original author(s) and the source, provide a link to the Creative Commons licence, and indicate if changes were made. The images or other third party material in this article are included in the article's Creative Commons licence, unless indicated otherwise in a credit line to the material. If material is not included in the article's Creative Commons licence and your intended use is not permitted by statutory regulation or exceeds the permitted use, you will need to obtain permission directly from the copyright holder. To view a copy of this licence, visit http://creativecommons.org/licenses/by/4.0/. 
Khan et al. [9] introduced the RK-iterative process in the setting of modular function spaces. Also, they studied fixed points of $\rho$-nonexpansive mappings in modular function spaces using $\Delta_{2}$ condition.

Moreover, Feng used the concept of w-compatible mappings to establish some new common coupled fixed point theorems for two hybrid pairs of mappings satisfying a symmetric type contractive condition in a partial metric space [6].

Benavides [2] revised some fixed point results for multivalued nonexpansive mappings in Banach and modular spaces. In addition, they found some new results depending either on the Opial modulus or on the Partington modulus in modular spaces.

On the other hand, in 2020, Faried et al., introduced the concepts of conjoint Fcontraction and conjoint F-contraction of Hardy-Rogers-type in the case of two multivalued mappings in regular modular metric space [5].

In this work, we generalize these concepts to the case of several multivalued Fproximinal mappings in regular modular metric space. Also, we establish a common fixed point theorems for several multivalued F-proximinal mappings in regular modular metric space. Finally, we give an application from our main results which establish the existence of the solution of integral equations.

\section{Preliminaries}

Throughout this paper, we use the following results.

Definition 2.1 ([7]) Let $X$ be a nonempty set. A function $\omega:(0, \infty) \times X \times X \rightarrow[0, \infty]$ is said to be a metric modular on $X$ if it satisfies, for all $x, y, z \in X$, the following conditions (we will write $\omega_{\lambda}(x, y)$ instead of $\omega(\lambda, x, y)$ ):

(1) $\omega_{\lambda}(x, y)=0$ for all $\lambda>0$ if and only if $x=y$,

(2) $\omega_{\lambda}(x, y)=\omega_{\lambda}(y, x)$ for all $\lambda>0$,

(3) $\omega_{\lambda+\mu}(x, y) \leq \omega_{\lambda}(x, z)+\omega_{\mu}(z, y)$ for all $\lambda, \mu>0$.

If instead of (3), we have the condition

(4) $\omega_{\lambda+\mu}(x, y) \leq \frac{\lambda}{\lambda+\mu} \omega_{\lambda}(x, z)+\frac{\mu}{\lambda+\mu} \omega_{\mu}(z, y)$ for all $\lambda, \mu>0$ and $x, y, z \in X$, then $\omega$ is called convex metric modular on $X$.

Also, if instead of (1), we have the condition

(5) $\omega_{\lambda}(x, x)=0$ for all $\lambda>0$, then $\omega$ is said to be a metric pseudomodular on $X$.

Definition 2.2 ([1]) Let $\omega$ be a pseudomodular on $X$. Fix $x_{0} \in X$, the two sets

$X_{\omega}\left(x_{0}\right)=\left\{x \in X: \lim _{\lambda \rightarrow \infty} \omega_{\lambda}\left(x, x_{0}\right)=0\right\}$ and $X_{\omega}^{*}\left(x_{0}\right)=\{x \in X: \exists \lambda=\lambda(x)>0$ such that $\left.\omega_{\lambda}\left(x, x_{0}\right)<\infty\right\}$ are said to be modular spaces generated by $x_{0}$.

The spaces $X_{\omega}\left(x_{0}\right)$ and $X_{\omega}^{*}\left(x_{0}\right)$ are metric spaces with the metrics $d_{\omega}(x, y)=\inf \{\lambda>$ $\left.0, \omega_{\lambda}(x, y)<\lambda\right\}$ and $d_{\omega}^{*}(x, y)=\inf \left\{\lambda>0, \omega_{\lambda}(x, y)<1\right\}$, respectively. For each $x, y \in X$ and $\lambda>0$, [1] defined $\omega_{\lambda^{+}}(x, y):=\lim _{\epsilon \rightarrow 0^{+}} \omega_{\lambda+\epsilon(x, y)}$ and $\omega_{\lambda-}(x, y):=\lim _{\epsilon \rightarrow 0^{+}} \omega_{\lambda-\epsilon(x, y)}$.

\section{Remark 2.3 ([3])}

(1) A metric modular $\omega$ on $X$ is nonincreasing with respect to $\lambda>0$. In fact, for any $x, y \in X$ and $0<\mu<\lambda$, we have $\omega_{\lambda}(x, y) \leq \omega_{\lambda-\mu}(x, x)+\omega_{\mu}(x, y)=\omega_{\mu}(x, y)$.

(2) $\omega_{\lambda^{+}}(x, y) \leq \omega_{\lambda}(x, y) \leq \omega_{\lambda^{-}}(x, y)$.

(3) If a metric modular $\omega$ on $X$ possesses a finite value for each $x, y \in X$ and $\omega_{\lambda}(x, y)=\omega_{\mu}(x, y)$ for all $\lambda, \omega>0$, then $d(x, y)=\omega_{\lambda}(x, y)$ is a metric on $X$. 
The following indexed objects $\omega$ are simple examples of (pseudo) modulars on a set $X$. Let $\lambda>0$ and $x, y \in X$, we have

Example 2.4 ([3])

$$
\begin{aligned}
\omega_{\lambda}^{a}(x, y)=\infty & \text { if } x \neq y, \\
=0 & \text { if } x=y,
\end{aligned}
$$

and if $(X, d)$ is a (pseudo)metric space with (pseudo) metric $d$, then we also have the following.

Example $2.5([3])$

$$
\omega_{\lambda}^{b}(x, y)=\frac{d(x, y)}{\varphi(\lambda)}
$$

for all $x, y \in X, \lambda>0$ where $\varphi:(0, \infty) \rightarrow(0, \infty)$ is any nondecreasing function.

Example $2.6([3])$

$$
\begin{aligned}
\omega_{\lambda}^{c}(x, y) & =\infty \quad \text { if } \lambda \leq d(x, y), \\
& =0 \quad \text { if } \lambda>d(x, y) .
\end{aligned}
$$

Example $2.7([3])$

$$
\begin{aligned}
\omega_{\lambda}^{d}(x, y)=\infty \quad \text { if } \lambda<d(x, y), \\
=0 \quad \text { if } \lambda \geq d(x, y) .
\end{aligned}
$$

Example $2.8([3])$ Let $(M, d)$ be a metric space and $X=M^{\mathbb{N}}$ be the set of all sequences $x: \mathbb{N} \rightarrow M$. Define $\omega_{\lambda}(x, y)$ by

$$
\omega_{\lambda}(x, y)=\sup _{n \in \mathbb{N}}\left(\frac{d(x(n), y(n))}{\lambda}\right)^{\frac{1}{n}}, \quad \lambda>0, x, y \in X .
$$

In general, if $\lim _{n \rightarrow \infty} \omega_{\lambda}\left(x_{n}, x\right)=0$ for some $\lambda>0$, then we may not have $\lim _{n \rightarrow \infty} \omega_{\lambda}\left(x_{n}\right.$, $x)=0$ for all $\lambda>0$. So, Chistyakov $[3,4]$ presented the following definition.

Definition 2.9 ([7]; Regular metric modular) A modular metric $\omega$ on $X$ is said to be regular if the following condition is satisfied:

$$
x=y \quad \text { if and only if } \omega_{\lambda}(x, y)=0 \quad \text { for some } \lambda>0 .
$$

This condition plays a significant role to ensure the existence of fixed point in modular metric space.

Definition 2.10 ([1]) Let $\omega$ be a metric modular on $X$ then 
(1) The sequence $\left\{x_{n}\right\}_{n \in \mathbb{N}}$ in $X$ is said to be $\omega$-convergent if and only if there exists $x \in X$ such that $\omega_{1}\left(x_{n}, x\right) \rightarrow 0$ as $n \rightarrow \infty$.

(2) The sequence $\left\{x_{n}\right\}_{n \in \mathbb{N}}$ in $X$ is said to be $\omega$-Cauchy if $\omega_{1}\left(x_{m}, x_{n}\right) \rightarrow 0$ as $m, n \rightarrow \infty$.

(3) A subset $D$ of $X$ is said to be $\omega$-complete if any $\omega$-Cauchy sequence in $D$ is a convergent sequence and its limit is in $D$.

(4) A subset $D$ of $X$ is said to be $\omega$-closed if $\omega$-limits of all $\omega$-convergent sequences of $D$ always belong to $D$.

(5) A subset $D$ of $X$ is said to be $\omega$-bounded if we have $\delta_{\omega}(D)=\sup \left\{\omega_{1}(x, y): x, y \in D\right\}<+\infty$.

(6) A subset $D$ of $X$ is said to be $\omega$-compact if for any $\left\{x_{n}\right\}_{n \in \mathbb{N}}$ in $D$ there exists a subsequence $\left\{x_{n_{k}}\right\}$ and $x \in D$ such that $\omega_{1}\left(x_{n_{k}}, x\right) \rightarrow 0$.

(7) $\omega$ is said to satisfy the Fatou property if and only if for any sequence $\left\{x_{n}\right\}_{n \in \mathbb{N}}$ in $X$ $\omega$-convergent to $x$, we have $\omega_{1}(x, y) \leq \liminf _{n \rightarrow \infty} \omega_{1}\left(x_{n}, y\right)$ for any $y \in X$.

Definition 2.11 ([7]; $\Delta_{2}$-condition) Let $(X, \omega)$ be a modular metric space and $\left\{x_{n}\right\}_{n \in \mathbb{N}}$ be a sequence in $X$. The metric modular $\omega$ is said to satisfy the $\Delta_{2}$-condition if $\lim _{n \rightarrow \infty} \omega_{\lambda}\left(x_{n}\right.$, $x)=0$ for some $\lambda>0$, then $\lim _{n \rightarrow \infty} \omega_{\lambda}\left(x_{n}, x\right)=0$ for all $\lambda>0$.

Definition 2.12 ([7]; $\Delta_{M}$-condition) Let $(X, \omega)$ be a modular metric space and $\left\{x_{n}\right\}_{n \in \mathbb{N}}$ be a sequence in $X$. The metric modular $\omega$ is said to satisfy the $\Delta_{M}$-condition if $\lim _{n \rightarrow \infty} \omega_{p}\left(x_{n+p}, x_{n}\right)=0$ or $(n \in \mathbb{N}, p>0)$ then $\lim _{n \rightarrow \infty} \omega_{\lambda}\left(x_{n+p}, x_{n}\right)=0$ for some $\lambda>0$.

\section{Multivalued F-contraction on modular metric space}

Throughout this paper, let $\mathcal{C B}(D)$ denote the set of all nonempty closed and bounded subsets of $D, \mathcal{C}(D)$ denotes the set of all nonempty closed subsets of $D$, and $\mathcal{C} \operatorname{Pr}(D)$ denotes the set of all closed proximinal subsets of $D$.

Let $A, B \in \mathcal{C} \operatorname{Pr}(D)$, we define the proximinal Hausdorff metric modular as follows:

$H_{\omega_{1}}(A, B):=\max \left\{\sup _{a \in A} \omega_{1}(a, B), \sup _{b \in B} \omega_{1}(b, A)\right\}$ where $\omega_{1}(a, B):=\inf _{b \in B} \omega_{1}(a, b)$.

Definition 3.1 ([7]) Let $F: \mathbb{R}^{+} \rightarrow \mathbb{R}$ be a function satisfying the following conditions:

(F1) $F$ is strictly increasing on $\mathbb{R}^{+}$.

(F2) For every sequence $\left\{s_{n}\right\}$ in $\mathbb{R}^{+}$, we have $\lim _{n \rightarrow \infty} s_{n}=0$ if and only if $\lim _{n \rightarrow \infty} F\left(s_{n}\right)=$ $-\infty$.

(F3) There exists a number $k \in(0,1)$ such that $\lim _{s \rightarrow 0^{+}} s^{k} F(s)=0$.

The family of all functions $F$ satisfying the conditions (F1)-(F3) is denoted by $\mathcal{F}$.

Definition 3.2 ([7]; F-contraction) Let $D$ be a nonempty $\omega$-bounded subset of a modular metric space $(X, \omega)$. For a fixed $F \in \mathcal{F}$ a multivalued mapping $T: D \rightarrow \mathcal{C B}(D)$ is called Fcontraction on $X$ if $\exists \tau \in \mathbb{R}^{+}$such that for any $x, y \in D$ with $y \in T x$ there exists $z \in T y$ such that $\omega_{1}(y, z)>0$ and the following inequality holds:

$$
\tau+F\left(\omega_{1}(y, z)\right) \leq F(M(x, y))
$$

where $M(x, y)=\max \left\{\omega_{1}(x, y), \omega_{1}(x, T x), \omega_{1}(y, T y), \omega_{1}(y, T x)\right\}$.

Definition 3.3 ([7]; F-contraction of Hardy-Rogers-type) Let $D$ be a nonempty $\omega$ bounded subset of a modular metric space $(X, \omega)$. A multivalued mapping $T: D \rightarrow \mathcal{C B}(D)$ 
is called an F-contraction of Hardy-Rogers-type if there exist $F \in \mathcal{F}$ and $\tau \in \mathbb{R}^{+}$such that

$$
2 \tau+F\left(H_{\omega}(T x, T y)\right) \leq F\left(\alpha \omega_{1}(x, y)+\beta \omega_{1}(x, T x)+\gamma \omega_{1}(y, T y)+L \omega_{1}(y, T x)\right) .
$$

Definition 3.4 ([8]; Proximinal) Let $E$ be a closed bounded subset of a Banach space $X$. The set $E$ is called proximinal in $X$ if for all $x \in X$ there is some $e \in E$ such that $\|x-e\|=$ $\inf \{\|x-y\|: y \in E\}$.

We will rewrite the following lemmas in the case of $\mathcal{C} \operatorname{Pr}(X)$.

Lemma 3.5 ([1]) Let $(X, \omega)$ be a modular metric space and $D$ be a nonempty subset of $X_{\omega}$. Let $A, B \in \mathcal{C} \mathcal{P} r(D)$ then for each $\epsilon>0$ and $a \in A$ there exists $b \in B$ such that

$$
\omega_{1}(a, b) \leq H_{1}(A, B)+\epsilon .
$$

Moreover, if $B$ is $\omega$-compact and $\omega$ satisfies the Fatou property, then for any $a \in A$ there exists $b \in B$ such that

$$
\omega_{1}(a, b) \leq H_{1}(A, B)
$$

Lemma 3.6 ([1]) Let $D$ be a nonempty subset of a modular metric space $(X, \omega)$. Assume that $\omega$ satisfies $\Delta_{2}$-condition and let $A_{n}$ be a sequence of sets in $\mathcal{C P r}(D)$ such that $\lim _{n \rightarrow \infty} H_{\omega_{1}}\left(A_{n}, A_{0}\right)=0$ where $A_{0} \in \mathcal{C} \mathcal{P} r(D)$. If $x_{n} \in A_{n}$ and $\lim _{n \rightarrow \infty} x_{n}=x_{0}$ then $x_{0} \in A_{0}$.

\section{Main results}

Now, we are ready to give our main results.

Definition 4.1 Let $D$ be a nonempty $\omega$-bounded subset of a modular metric space $(X, \omega)$. For fixed $F \in \mathcal{F}$, we say that multivalued mappings $T_{i}, T_{i+1}: D \rightarrow \mathcal{C} \mathcal{P r}(D)$ form a conjoint F-proximinal contraction on $X$ for $i=1,2, \ldots, k$. If for $0<q<1$ and all $x, y \in D$ such that $H_{\omega_{1}}\left(T_{i} x, T_{i+1} y\right)>0$ the following inequality holds:

$$
0<\inf \left\{F\left(M_{T_{i}, T_{i+1}}(x, y)\right)-F\left(H_{\omega_{1}}\left(T_{i} x, T_{i+1} y\right)\right)\right\}
$$

and $M_{T_{i}, T_{i+1}}(x, y)=q\left(\max \left\{\omega_{1}(x, y), \omega_{1}\left(y, T_{i} x\right), \omega_{1}\left(T_{i} x, x\right), \omega_{1}\left(T_{i+1} y, y\right)\right\}\right)$.

Theorem 4.2 Let D be a nonempty $\omega$-bounded and $\omega$-complete subset of a modular metric space $(X, \omega)$. Assume that $\omega$ is a regular modular satisfying $\Delta_{M}$ and $\Delta_{2}$-conditions. If $T_{i}, T_{i+1}: D \rightarrow \mathcal{C P} r(D)$ form continuous conjoint F-proximinal contractions for each $i=1,2, \ldots, k$ then they have a unique common fixed point for each $i=1,2, \ldots, k$. In other words, there exists $u \in D$ such that $u \in T_{1} u, u \in T_{2} u, \ldots$ and $u \in T_{k} u$.

Proof Since $T_{1}$ and $T_{2}$ form a continuous conjoint F-proximinal contraction, there exists a unique common fixed point $u_{1}$ between $T_{1}$ and $T_{2}$ or $u_{1} \in T_{1} u_{1}$ and $u_{1} \in T_{2} u_{1}$. Similarly, $T_{2}$ and $T_{3}$ form a continuous conjoint F-proximinal contraction; then there exists a unique common fixed point $u_{2}$ between $T_{2}$ and $T_{3}$ or $u_{2} \in T_{2} u_{2}$ and $u_{2} \in T_{3} u_{2}$.

Now we will show that $u_{1}=u_{2}$. 
Assume contrarily that $u_{1} \neq u_{2}$ and we have

$$
0<\inf \left\{F\left(M_{T_{1}, T_{2}}\left(u_{1}, u_{2}\right)\right)-F\left(H_{\omega_{1}}\left(T_{1} u_{1}, T_{2} u_{2}\right)\right)\right\} .
$$

Or

$$
F\left(H_{\omega_{1}}\left(T_{1} u_{1}, T_{2} u_{2}\right)\right)+\tau \leq F\left(M_{T_{1}, T_{2}}\left(u_{1}, u_{2}\right)\right), \quad \text { for some } \tau>0 \text {, }
$$

i.e.

$$
\begin{aligned}
H_{\omega_{1}}\left(T_{1} u_{1}, T_{2} u_{2}\right) & <M_{T_{1}, T_{2}}\left(u_{1}, u_{2}\right) \\
& =q\left(\max \left\{\omega_{1}\left(u_{1}, u_{2}\right), \omega_{1}\left(u_{2}, T_{1} u_{1}\right), \omega_{1}\left(T_{1} u_{1}, u_{1}\right), \omega_{1}\left(T_{2} u_{2}, u_{2}\right)\right\}\right) \\
& =q\left(\max \left\{\omega_{1}\left(u_{1}, u_{2}\right), \omega_{1}\left(u_{2}, T u_{1}\right)\right\}\right) .
\end{aligned}
$$

Since $\omega_{1}\left(u_{1}, u_{2}\right) \leq H_{\omega_{1}}\left(T_{1} u_{1}, T_{2} u_{2}\right)$,

$$
\omega_{1}\left(u_{1}, u_{2}\right)<q\left(\max \left\{\omega_{1}\left(u_{1}, u_{2}\right), \omega_{1}\left(u_{2}, T_{1} u_{1}\right)\right\}\right) .
$$

Since $u_{1} \in T_{1} u_{1}$ then $\omega_{1}\left(u_{2}, T_{1} u_{1}\right) \leq \omega_{1}\left(u_{1}, u_{2}\right)$, i.e.

$$
\max \left\{\omega_{1}\left(u_{1}, u_{2}\right), \omega_{1}\left(u_{2}, T_{1} u_{1}\right)\right\}=\omega_{1}\left(u_{1}, u_{2}\right) .
$$

Then from Eqs. (4.2) and (4.3), we get

$$
\omega_{1}\left(u_{1}, u_{2}\right)<q \omega_{1}\left(u_{1}, u_{2}\right) .
$$

Then $u_{1}=u_{2}$, which gives a contradiction with $u_{1} \neq u_{2}$ or $\omega_{1}\left(u_{1}, u_{2}\right) \neq 0$. So $u_{1}=u_{2}=u$ such that $u$ is a unique common fixed point for $T_{1}, T_{2}$ and $T_{3}$ or $u \in T_{1} u, u \in T_{2} u$ and $u \in T_{3} u$

By repeating this procedure for $T_{2}, T_{3}$ and $T_{4}$ we can deduce that there exists a unique common fixed point $v \in D$ for $T_{2}, T_{3}$ and $T_{4}$ such that $v \in T_{2} v, v \in T_{3} v$ and $v \in T_{4} v . u$ is unique for $T_{1}, T_{2}$ and $T_{3}$ and $v$ is unique for $T_{2}, T_{3}$ and $T_{4}$. Now

$$
\begin{aligned}
\omega_{1}(u, v) & \leq H_{\omega_{1}}\left(T_{2} u, T_{3} v\right) \\
& <M_{T_{1}, T_{2}}(u, v) \\
& =q\left(\max \left\{\omega_{1}(u, v), \omega_{1}\left(v, T_{2} u\right), \omega_{1}\left(T_{2} u, u\right), \omega_{1}\left(T_{3} v, v\right)\right\}\right) \\
& =q\left(\max \left\{\omega_{1}(u, v), \omega_{1}\left(v, T_{2} u\right)\right\}\right) .
\end{aligned}
$$

Since $u \in T_{2} u$ we have $\omega_{1}\left(v, T_{2} u\right) \leq \omega_{1}(u, v)$.

Then

$$
\omega_{1}(u, v)<q \omega_{1}(u, v)
$$

which gives a contradiction. Therefore, $\omega_{1}(u, v)=0$ and $u=v$.

We conclude that there exists a unique common fixed point for $T_{1}, T_{2}, T_{3}$ and $T_{4}$. Finally, we see that $u$ is a common fixed point for $T_{1}, T_{2}, \ldots$, and $T_{k}$. 
Definition 4.3 Let $D$ be a nonempty bounded subset of a modular metric space $(X, \omega)$. The multivalued mappings $T_{i}, T_{i+1}: D \rightarrow \mathcal{C} \mathcal{P r}(D)$ are called conjoint F-proximinal contraction of Hardy-Rogers-type on $X$ if there exists $F \in F$, and

$$
\begin{aligned}
0< & \inf \left\{F \alpha \omega_{1}(x, y)+\beta \omega_{1}\left(x, T_{i} x\right)+\gamma \omega_{1}\left(y, T_{i+1} y\right)\right. \\
& \left.\left.+L \omega_{1}\left(y, T_{i} x\right)\right)-F\left(H_{\omega_{1}}\left(T_{i} x, T_{i+1} y\right)\right)\right\}
\end{aligned}
$$

for all $x, y \in D$ with $H_{\omega_{1}}\left(T_{i} x, T_{i+1} y\right)>0$, where $\alpha, \beta, \gamma, L \geq 0, \alpha+\beta+\gamma=1, \gamma<1, \beta+L<1$ and $\alpha+L<1$ for each $i=1,2, \ldots, k$.

Theorem 4.4 Let $D$ be a nonempty $\omega$-bounded and $\omega$-complete subset of a modular metric space $(X, \omega)$. Assume that $\omega$ is a regular modular satisfying $\Delta_{M}$-and $\Delta_{2}$-conditions and $T_{i}, T_{i+1}: D \rightarrow \mathcal{C} \mathcal{P r}(D)$ are continuous conjoint F-proximinal contractions of Hardy-Rogerstype on $X$ for each $i=1,2, \ldots, k$. Consequently, they have a common fixed point $u \rightarrow D$ such that $u \rightarrow T_{1} u, u \rightarrow T_{2} u, \ldots$ and $u \rightarrow T_{k} u$.

Proof Since $T_{1}$ and $T_{2}$ be continuous conjoint F-proximinal contraction of HardyRogers-type on $X$, there exists $u_{1} \rightarrow D$ such that $u_{1} \rightarrow T_{1} u_{1}, u_{1} \rightarrow T_{2} u_{1}$. Also, let $T_{2}$ and $T_{3}$ be continuous conjoint F-proximinal contraction of Hardy-Rogers-type on $X$; then there exists $u_{2} \rightarrow D$ such that $u_{2} \rightarrow T_{2} u_{2}, u_{2} \rightarrow T_{3} u_{2}$.

Now we will show that $u_{1}=u_{2}$.

Since $T_{1}$ and $T_{2}$ are continuous conjoint F-proximinal contraction of Hardy-Rogerstype on $X$,

$$
\begin{aligned}
0< & \inf \left\{F \left(\alpha \omega_{1}\left(u_{1}, u_{2}\right)+\beta \omega_{1}\left(u_{1}, T_{1} u_{1}\right)+\gamma \omega_{1}\left(u_{2}, T_{2} u_{2}\right)\right.\right. \\
& \left.\left.+L \omega_{1}\left(u_{2}, T_{1} u_{1}\right)\right)-F\left(H_{\omega_{1}}\left(T_{1} u_{1}, T_{2} u_{2}\right)\right)\right\} .
\end{aligned}
$$

Or

$$
\begin{aligned}
F\left(H_{\omega_{1}}\left(T_{1} u_{1}, T_{2} u_{2}\right)\right)+\tau \leq & F\left(\alpha \omega_{1}\left(u_{1}, u_{2}\right)+\beta \omega_{1}\left(u_{1}, T_{1} u_{1}\right)+\gamma \omega_{1}\left(u_{2}, T_{2} u_{2}\right)\right. \\
& \left.+L \omega_{1}\left(u_{2}, T_{1} u_{1}\right)\right)
\end{aligned}
$$

for some $\tau>0$, i.e.,

$$
H_{\omega_{1}}\left(T_{1} u_{1}, T_{2} u_{2}\right)<\alpha \omega_{1}\left(u_{1}, u_{2}\right)+\beta \omega_{1}\left(u_{1}, T_{1} u_{1}\right)+\gamma \omega_{1}\left(u_{2}, T_{2} u_{2}\right)+L \omega_{1}\left(u_{2}, T_{1} u_{1}\right) .
$$

Or

$$
\omega_{1}\left(u_{1}, u_{2}\right)<\alpha \omega_{1}\left(u_{1}, u_{2}\right)+\beta \omega_{1}\left(u_{1}, T_{1} u_{1}\right)+\gamma \omega_{1}\left(u_{2}, T_{2} u_{2}\right)+L \omega_{1}\left(u_{2}, T_{1} u_{1}\right) .
$$

Since $u_{1} \in T_{1} u_{1}, u_{1} \in T_{2} u_{1}, u_{2} \in T_{2} u_{2}$ and $u_{2} \in T_{3} u_{2}$

$$
\begin{aligned}
\omega_{1}\left(u_{1}, u_{2}\right) & <\alpha \omega_{1}\left(u_{1}, u_{2}\right)+L \omega_{1}\left(u_{2}, T_{1} u_{1}\right) \\
& \leq \alpha \omega_{1}\left(u_{1}, u_{2}\right)+L \omega_{1}\left(u_{2}, u_{1}\right) \\
& =(\alpha+L) \omega_{1}\left(u_{2}, u_{1}\right),
\end{aligned}
$$

which gives a contradiction since $\alpha+L<1$. 
Hence, $u_{1}=u_{2}=u$.

Therefore, there exists $u \in D$ such that $u$ is a common fixed point for $T_{1}, T_{2}$ and $T_{3}$.

By repeating this procedure for $T_{2}, T_{3}$ and $T_{4}$ we deduce that $v$ is a common fixed point for $T_{2}, T_{3}$ and $T_{4}$.

Now we will show that $u=v$. Assume contrarily that $u \neq v$; hence for $u \in T_{2} u$ and $v \in T_{3} v$ we have

$$
\begin{aligned}
\omega_{1}(u, v) & <H \omega_{1}\left(T_{2} u, T_{3} v\right) \\
& <\alpha \omega_{1}(u, v)+\beta \omega_{1}\left(u, T_{2} u\right)+\gamma \omega_{1}\left(v, T_{3} v\right)+L \omega_{1}\left(v, T_{2} u\right) \\
& \leq \alpha \omega_{1}(u, v)+L \omega_{1}(u, v) \\
& \leq(\alpha+L) \omega_{1}(u, v) .
\end{aligned}
$$

Since $\alpha+L<1$ we have $\omega_{1}(u, v)=0$ and $u=v$. Therefore, $u$ is a common fixed point for $T_{1}, T_{2}, T_{3}$ and $T_{4}$. Finally, we get a common fixed point for $T_{1}, T_{2}, \ldots$, and $T_{k}$.

\section{Application to integral equations}

In this section, we give an application of Theorem 4.2 to Volterra-type integral equations. Let $\left(C[0, a],\|\cdot\|_{\tau}\right)$ be a Banach space where $C[0, a]$ is the set of all continuous functions on $[0, a]$. Consider the integrals

$$
u_{i}(t)=\int_{0}^{t} K_{i}(t, s, u(s)) d s+f_{i}(t)
$$

for all $t \in[0, a]$ and $i=1,2, \ldots, k$. We take $u_{i} \in C[0, a]$ with the norm

$$
\left\|u_{i}\right\|_{\tau}=\max _{t \in[0, a]}\left|u_{i}(t) e^{-\tau t}\right|
$$

for arbitrary $\tau>0$ and the metric

$$
\omega_{\lambda}\left(u_{i}, u_{i+1}\right)=\frac{1}{\lambda}\left\|u_{i}-u_{i+1}\right\|_{\tau}=\frac{1}{\lambda} \max _{t \in[0, a]}\left|\left(u_{i}(t)-u_{i+1}(t)\right) e^{-\tau t}\right|
$$

for all $u_{i}, u_{i+1} \in C[0, a]$.

Now we will prove the following theorem to ensure the existence of the solution of the system of integral equations.

Theorem 5.1 Consider $K_{i}:[0, a] \times[0, a] \times \mathbb{R} \rightarrow \mathbb{R}, f_{i}:[0, a] \rightarrow \mathbb{R}$ to be continuous and

$$
T_{i}: C[0, a] \rightarrow \mathcal{C} \operatorname{Pr}(C[0, a]) \text { as }
$$

$$
T_{i} u_{i}(t)=\left(\int_{0}^{t} K_{i}\left(t, s, u_{i}(s)\right) d s+f_{i}(t)\right) e^{-\frac{n}{i} t}
$$

for every $n \in \mathbb{N} \cup\{0\}$ and $i=1,2, \ldots, k$. If there exists $\tau>1$, such that

$$
\begin{aligned}
& \sup _{n, m \in \mathbb{N} \cup\{0\}}\left\{\left|K_{i}\left(t, s, u_{i}(s)\right) e^{-\frac{n}{i} t}-K_{i+1}\left(t, s, u_{i+1}(s)\right) e^{-\frac{m}{i+1} t}\right|+\left|f_{i}(t) e^{-\frac{n}{i} t}-f_{i+1}(t) e^{-\frac{m}{i+1} t}\right|\right\} e^{-\tau t} \\
& \leq \tau e^{-\tau}\left|M_{T_{i}, T_{i+1}}\left(u_{i}(t), u_{i+1}(t)\right)\right|
\end{aligned}
$$


for some $t \in[0, a]$, for every $n, m \in \mathbb{N} \cup\{0\}, u_{i} \in C[0, a]$ and $i=1,2, \ldots, k$, then the system of integral equations (5.2) has a solution.

Proof Choosing $x^{*}$ and $y^{*}$ to be among the best approximations of $T_{i+1} u_{i+1}(t)$ and $T_{i} u_{i}(t)$, we have

$$
\begin{aligned}
H\left(T_{i} u_{i}(t), T_{i+1} u_{i+1}(t)\right) & =\max \left\{\sup _{x \in T_{i} u_{i}(t)} \omega_{1}\left(x, T_{i+1} u_{i+1}(t)\right), \sup _{y \in T_{i+1} u_{i+1}(t)} \omega_{1}\left(y, T_{i} u_{i}(t)\right)\right\} \\
& =\max \left\{\sup _{x \in T_{i} u_{i}(t)} \omega_{1}\left(x, x^{*}\right), \sup _{y \in T_{i+1} u_{i+1}(t)} \omega_{1}\left(y, y^{*}\right)\right\} \\
& \leq \sup _{x \in T_{i} u_{i}(t), y \in T_{i+1} u_{i+1}(t)} \omega_{1}(x, y)
\end{aligned}
$$

but $\sup _{x \in T_{i} u_{i}(t), y \in T_{i+1} u_{i+1}(t)} \omega_{1}(x, y) \leq \sup _{n, m \in \mathbb{N} \cup\{0\}}\left\{\int_{0}^{t} \mid K_{i}\left(t, s, u_{i}(s)\right) e^{-\frac{n}{i} t}-K_{i+1}\left(t, s, u_{i+1}(s)\right) \times\right.$ $\left.e^{-\frac{m}{i+1} t}|d s+| f_{i}(t) e^{-\frac{n}{i} t}-f_{i+1}(t) e^{-\frac{m}{i+1} t} \mid d s\right\} e^{-\tau t}$ so

$$
\begin{aligned}
H\left(T_{i} u_{i}(t), T_{i+1} u_{i+1}(t)\right) & \leq \tau e^{-\tau} \int_{0}^{t}\left|M_{T_{i}, T_{i+1}}\left(u_{i}(t), u_{i+1}(t)\right)\right| e^{-\tau s} e^{\tau s} d s \\
& \leq\left\|M_{T_{i}, T_{i+1}}\left(u_{i}(t), u_{i+1}(t)\right)\right\|_{\tau} \tau e^{-\tau} \int_{0}^{t} e^{\tau s} d s \\
& =\left\|M_{T_{i}, T_{i+1}}\left(u_{i}(t), u_{i+1}(t)\right)\right\|_{\tau} \tau e^{-\tau} \frac{e^{\tau t}}{\tau} \\
& =\left\|M_{T_{i}, T_{i+1}}\left(u_{i}(t), u_{i+1}(t)\right)\right\|_{\tau} e^{-\tau} e^{\tau t}
\end{aligned}
$$

for any $t \in[0, a]$, for every $n, m \in \mathbb{N} \cup\{0\}, u_{i} \in C[0, a]$ and $i=1,2, \ldots, k$. Dividing by $e^{\tau t}$, we get

$$
H\left(T_{i} u_{i}(t), T_{i+1} u_{i+1}(t)\right) e^{-\tau t} \leq e^{-\tau}\left\|M_{T_{i}, T_{i+1}}\left(u_{i}(t), u_{i+1}(t)\right)\right\|_{\tau} .
$$

So,

$$
\left\|H\left(T_{i} u_{i}(t), T_{i+1} u_{i+1}(t)\right)\right\|_{\tau} \leq e^{-\tau}\left\|M_{T_{i}, T_{i+1}}\left(u_{i}(t), u_{i+1}(t)\right)\right\|_{\tau} .
$$

This implies that

$$
\tau+\ln \left\|H\left(T_{i} u_{i}(t), T_{i+1} u_{i+1}(t)\right)\right\|_{\tau} \leq\left\|M_{T_{i}, T_{i+1}}\left(u_{i}(t), u_{i+1}(t)\right)\right\|_{\tau} .
$$

So, all the conditions of Theorem 4.2 are satisfied if $F(\alpha)=\ln \alpha$. Hence there exists $r \in$ $C[0, a]$ such that

$$
\begin{aligned}
& r(t) \in T_{1} r(t)=\left\{\left(\int_{0}^{t} K_{1}(t, s, r(s)) d s+f_{1}(t)\right) e^{-n t}\right\}, \\
& r(t) \in T_{2} r(t)=\left\{\left(\int_{0}^{t} K_{2}(t, s, r(s)) d s+f_{2}(t)\right) e^{-\frac{n}{2} t}\right\}, \ldots, \quad \text { and } \\
& r(t) \in T_{k} r(t)=\left\{\left(\int_{0}^{t} K_{k}(t, s, r(s)) d s+f_{k}(t)\right) e^{-\frac{n}{k} t}\right\} .
\end{aligned}
$$


Finally, there exist $K_{i}, f_{i}, n_{1}, n_{2}, \ldots, n_{k} \in \mathbb{N} \cup\{0\}$ such that $r(t)$ is a solution of the system of integral equations given in (5.2) for $i=1,2, \ldots, k$.

\section{Conclusion}

In this paper we presented the new concepts of conjoint F-contraction and conjoint Fcontraction of Hardy-Rogers-type to the case of several multivalued F-proximinal mappings in regular modular metric space. Also, we used these concepts to found a common fixed point theorems for several multivalued F-proximinal mappings in regular modular metric space. The solution of integral equations was obtained by employing the condition of conjoint F-contraction for several multivalued F-proximinal mappings in regular modular metric space.

\section{Acknowledgements}

We are thankful to the reviewers for their careful reading and valuable comments, which considerably improved this paper.

\section{Funding}

The authors were supported financially while writing this paper only from their own personal sources.

\section{Abbreviations}

MVM, multivalued mappings.

Availability of data and materials

Data sharing is not applicable to this paper.

Competing interests

The authors declare that they have no competing interests.

\section{Authors' contributions}

The authors contributed equally and approved the final draft of this manuscript.

\section{Publisher's Note}

Springer Nature remains neutral with regard to jurisdictional claims in published maps and institutional affiliations.

Received: 31 March 2021 Accepted: 23 June 2021 Published online: 27 July 2021

\section{References}

1. Abdou, A.A.N., Khamsi, M.A.: Fixed points of multivalued contraction mappings in modular metric spaces. Fixed Point Theory Appl. 2014, 249 (2014)

2. Benavides, T.D., Ramírez, P.L.: Fixed points for multivalued non-expansive mappings. Appl. Set-Valued Anal. Optim. 2 143-157 (2020)

3. Chaistyakov, V.V.: Modular metric spaces, I: basic concepts. Nonlinear Anal. 72, 1-14 (2010)

4. Chaistyakov, V.V.: Modular metric spaces. II. Application to superposition operators. Nonlinear Anal. 72, 15-30 (2010)

5. Faried, N., Abd El Ghaffar, H., Hamdy, S.: Common fixed point for two multivalued mappings on proximinal sets in regular modular metric space. Pure Appl. Math. J. 9(4), 74-83 (2020)

6. Gu, F., Shatanawi, W.: Some new results on common coupled fixed points of two hybrid pairs of mappings in partial metric spaces. J. Nonlinear Funct. Anal. 2019, Article ID 13 (2019)

7. Jain, D., Padcharoen, A., Kumam, P., Gopal, D.: A new approach to study fixed point of multivalued mappings in modular metric spaces and applications. Mathematics 4, 51 (2016)

8. Khalil, R., Mater, N.: Every strongly remotal subset in Banach spaces is a singleton. Br. J. Math. Comput. Sci. 5, 28-34 (2015)

9. Khan, S.H., Fukhar-ud-din, H.: Approximating fixed points of $\rho$-nonexpansive mappings by RK-iterative process in modular function spaces. J. Nonlinear Var. Anal. 3, 107-114 (2019)

10. Khan, S.U., Ghaffar, A., Ullah, Z., Rasham, T., Arshad, M.: On fixed point theorems via proximinal maps in partial metric spaces with applications. J. Math. Anal. 9, 28-39 (2018)

11. Mongkolkeha, C., Kumam, P.: Common fixed points for generalized weak contraction mappings in modular metric spaces. Sci. Math. Jpn. e-2012, 117-127 (2012)

12. Nadler, S.B.: Multi-valued contraction mappings. Pac. J. Math. 30, 475-488 (1969)

13. Rahimpoor, H., Ebadian, A., Gordji, M.E., Zohri, A.: Common fixed point theorem in modular metric spaces. Int. J. Pure Appl. Math. 99(3), 373-383 (2015)

14. Sgroi, M., Vetro, C.: Multi-valued F-contractions and the solution of certain functional and integral equations. Filomat 27, 1259-1268 (2013)

15. Wardowski, D.: Fixed points of new type of contractive mappings in complete metric space. Fixed Point Theory Appl. 2012, 94 (2012) 